\title{
Article \\ Performance of Micro-Scale Transmission \& Reception Diversity Schemes in High Throughput Satellite Communication Networks
}

\author{
Apostolos Z. Papafragkakis (D), Charilaos I. Kouroriorgas (D) and Athanasios D. Panagopoulos * \\ Radio and Satellite Communications Group, Division of Information Transmission Systems and Materials \\ Technology, School of Electrical and Computer Engineering, National Technical University of Athens, Iroon \\ Polytechniou 9, GR-157 80 Athens, Greece; apapafrag@mail.ntua.gr (A.Z.P.); harkour@mail.ntua.gr (C.I.K.) \\ * Correspondence: thpanag@ece.ntua.gr; Tel.: +30-2107723842
}

Citation: Papafragkakis, A.Z.; Kouroriorgas, C.I.; Panagopoulos, A.D. Performance of Micro-Scale Transmission \& Reception Diversity Schemes in High Throughput Satellite Communication Networks. Electronics 2021, 10, 2073. https:// doi.org/10.3390/electronics10172073

Academic Editor: Mauro Tropea

Received: 30 July 2021

Accepted: 23 August 2021

Published: 27 August 2021

Publisher's Note: MDPI stays neutral with regard to jurisdictional claims in published maps and institutional affiliations.

Copyright: () 2021 by the authors. Licensee MDPI, Basel, Switzerland. This article is an open access article distributed under the terms and conditions of the Creative Commons Attribution (CC BY) license (https:// creativecommons.org/licenses/by/ $4.0 /)$.
Abstract: The use of $\mathrm{Ka}$ and $\mathrm{Q} / \mathrm{V}$ bands could be a promising solution in order to accommodate higher data rate, interactive services; however, at these frequency bands signal attenuation due to the various atmospheric phenomena and more particularly due to rain could constitute a serious limiting factor in system performance and availability. To alleviate this possible barrier, short- and large-scale diversity schemes have been proposed and examined in the past; in this paper a micro-scale site diversity system is evaluated in terms of capacity gain using rain attenuation time series generated using the Synthetic Storm Technique (SST). Input to the SST was 4 years of experimental rainfall data from two stations with a separation distance of $386 \mathrm{~m}$ at the National Technical University of Athens (NTUA) campus in Athens, Greece. Additionally, a novel multi-dimensional synthesizer based on Gaussian Copulas parameterized for the case of multiple-site micro-scale diversity systems is presented and evaluated. In all examined scenarios a significant capacity gain can be observed, thus proving that micro-scale site diversity systems could be a viable choice for enterprise users to increase the achievable data rates and improve the availability of their links.

Keywords: micro-scale site diversity; MIMO; SIMO; MISO; satellite; rain rate; attenuation; Synthetic Storm Technique; Gaussian copulas; outage capacity

\section{Introduction}

The use of high data rate satellite services has recently become a necessity given the vast number of new or planned applications which will be in high demand in the upcoming $5 \mathrm{G}$ era [1,2]. Satellite communication will play an important role in the $5 \mathrm{G}$ vision $[3,4]$ by either providing back-hauling services or offering internet services in under-served areas; satellite back-haul not only allows for connectivity extension at remote locations but also serves as the prime solution for use cases such as emergency response, network backup and Internet of Things (IoT) applications.

In order to increase the provided data rates in an efficient, cost-effective manner and taking into consideration the spectrum scarcity problem [5,6], next generation satellite communication systems are migrating to the $\mathrm{Ka}$ and $\mathrm{Q} / \mathrm{V}$ bands and are expected to make extensive use of link adaptation strategies combined with multi-beam satellites and/or other relevant techniques [7-14]. Signal propagation at Ka-band frequencies and above is, nevertheless, highly affected by the various atmospheric phenomena causing degradation of the overall system performance. In particular, precipitation, clouds, atmospheric gases and turbulence can introduce significant losses into the system, with rain being the dominant fading mechanism $[15,16]$. In order to compensate for rain attenuation, Fading Mitigation Techniques are required (FMTs) [17]. FMTs include link adaptation techniques such as uplink power control and Adaptive Coding and Modulation (ACM) schemes. However, the most effective FMT is the use of spatial diversity, e.g., site diversity, since such a technique exploits the spatial variability of the precipitation medium. 
In site diversity scenarios, one or more ground stations communicate with the same satellite and therefore, the probability that all of the links simultaneously experience severe attenuation is smaller than having a single station. In general, the gain of a site diversity system increases with increasing the distance between the stations. Nonetheless, a considerable gain can be observed even when the distance between the stations is appreciably small, such as in [18-21]. Such systems can be employed in enterprise scenarios such as (but not limited to) company headquarters, sports events, concerts or media events. In such cases, the stations can be deployed around the company buildings, a stadium, or a concert hall forming a micro-scale site diversity scheme. In cases where multiple stations are used, Multiple Input Multiple Output (MIMO) techniques could also be employed to improve the system's performance even further. Regarding the use of MIMO techniques in satellite communications, selection diversity is mostly preferred and implemented, however, by exploiting the knowledge of the channel conditions, the system performance can be further enhanced [22].

In the context of satellite communications, due to the use of massive, very costly Gateways (GWs) used for data forwarding, it is more likely to have multiple stations at the user premises than at the GW sites [23-25]; therefore, at frequency bands such as Ka-band and above, the use of Multiple Input Single Output (MISO) and Single Input Multiple Output (SIMO) techniques with multiple antenna configurations used at the user terminals seem a potential solution to mitigate the various non-desirable propagation effects. In a MISO system the uplink can be enhanced, while in a SIMO system the downlink, respectively. Multi-path effects at such high frequencies are insignificant and thus, in order to exploit the MIMO principle the benefits arising from the spatial inhomogeneity of the rainfall are more relevant. According to [22], system capacity can be increased for both SIMO and MISO cases provided that perfect knowledge of the channel state is available at the receiver and transmitter, respectively. The Channel State Information (CSI) from the user terminals can be extracted either through beacon signals and/or radiometers [26,27], or by exploiting the pilot symbols used in the transmitted frames [28,29].

Prior to designing a communication system with built-in FMTs, multi-dimensional rain attenuation time series synthesizers are essential in order to capture the spatio-temporal characteristics of the propagation effects due to rain. In [30], a multi-dimensional time series synthesizer based on stochastic differential equations has been presented, while in [31] a filter-based method. Both models are based on the main assumptions of the MassengBakken model [32], i.e., that rain attenuation follows the log-normal distribution and the Gaussian underlined process has an exponentially decreasing auto-correlation function; both models use the Pearson's correlation coefficient to model the spatial correlation. In [33], another model is presented in which the Numerical Weather Prediction (NWP) analysis products are used; starting from rain rate, rain attenuation time series are generated in maps and therefore correlation is induced. Nevertheless, in the latter methodology data resolution is small rendering it inappropriate for use in micro-scale systems. In [34], a new approach for modeling joint statistics of rain attenuation in site-diversity systems based on Gaussian copula functions [35] has been presented, while in [36], the same copula functions are used to model the joint temporal statistics of rain attenuation through the generation of rain attenuation time series for single links. The advantages of using copula functions are: (a) there is no need to model the single-link rain attenuation statistics with a particular distribution and (b) rain attenuation between multiple links is not assumed to be linearly correlated in order to model their spatial dependence.

In this paper, the use of a micro-scale site diversity scheme for increasing the system capacity is investigated. Taking advantage of four years of available rainfall measurements for two locations within the National Technical University of Athens (NTUA) campus, rain attenuation time series are obtained using the Synthetic Storm Technique (SST) [37] and the outage capacity is then evaluated for a micro-scale dual-site diversity system. The SST is used due its highly accurate method of capturing the cumulative statistics of rain attenuation [38]. Moreover, a multi-dimensional time series synthesizer based on Gaussian copulas 
is proposed for generating rain attenuation time series at multiple spatially separated links. The synthesizer is based on the assumption that in the spatial and the temporal domain the joint distribution of rain attenuation can be modeled through Gaussian copulas. The spatial parameter of the copula is calculated from the rain attenuation time series derived using SST in order for the synthesizer to allow the evaluation of multi-site micro-scale diversity systems (e.g., triple and quadruple). Experimental campaigns for micro-scale site diversity scenarios have to be conducted in order to validate the presented results.

The contributions of the paper are the following: (a) a micro-scale site diversity system is evaluated using rain rate time series and the achieved gain is observed, (b) a novel multi-dimensional rain attenuation time series synthesizer for multiple slant paths based on copula functions is proposed (c) a new spatial correlation coefficient for the rainfall medium across small distances is given (d) the performance of multi-site micro-scale transmission and reception schemes operating at Ka and Q band as MISO and SIMO schemes are investigated for the first time in the literature.

The remainder of the paper is organized as follows: in Section 2, the system model and the expressions for the calculation of capacity for MISO and SIMO systems are given. In Section 3, the 4-year rainfall data-bank of NTUA is used for the evaluation of a dual-site micro-scale diversity system. In Section 4, a multi-dimensional copulas synthesizer is presented and used for the evaluation of micro-scale site diversity systems with multiple stations. In Section 5, the paper concludes summarizing the used methodology and commenting on the obtained results.

\section{System Model}

In this section, the system model used for the evaluation of micro-scale site diversity systems is presented. Both dual- and multi-site micro-scale diversity systems are examined. Figure 1 presents the system geometries consisting of a conventional single station scheme (Figure 1a) as well as three micro-scale site diversity systems for two, three and four ground stations, respectively, in Figure 1b-d. The system is assumed to operate at Ka-band and above with highly directive antennas; the multiple links do not interfere with each other, there is always Line-of-Sight (LOS) and any attenuation is caused due to the signal propagating through the atmosphere with rain being the dominant fading mechanism.

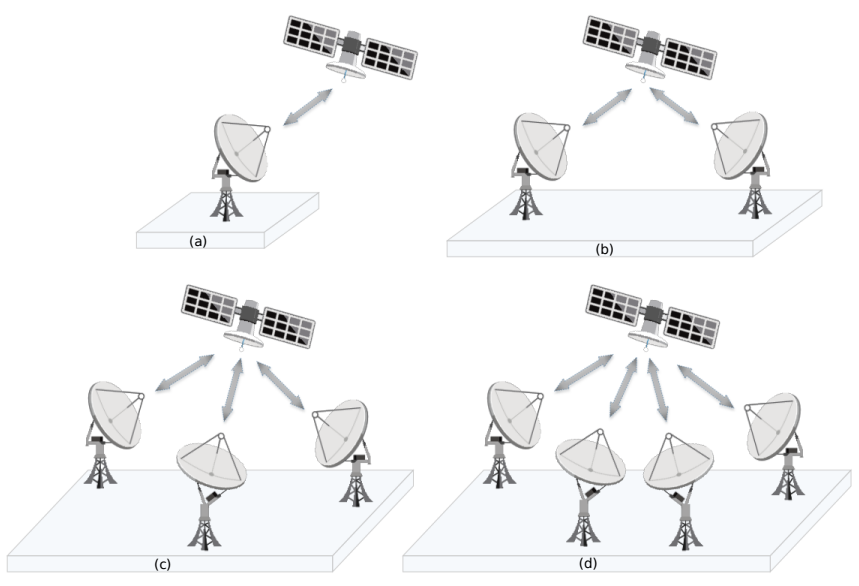

Figure 1. System configurations for (a) single-link systems, (b) dual-site micro-scale diversity systems, (c) triple-site micro-scale diversity systems and (d) quad-site micro-scale diversity systems.

In the system examined herein, perfect knowledge of the channel conditions at both the transmitting and the receiving end of the stations is assumed (i.e., the induced rain attenuation at the slant paths); the stations are considered to be identical and interconnected with one another. As already mentioned in the introduction, in order to acquire the channel state the ground stations are either equipped with beacon receivers at the 
operating frequency (or close enough) or estimate the channel using the pilot symbols of the frame. Considering that the links operate at Ka-band frequencies and above, the channel conditions shall remain constant within the Round-Trip Time (RTT) delay, i.e., approximately $500 \mathrm{~ms}$ for a GEO link. A highly appreciated metric used in the design of satellite communication systems is the outage capacity, i.e., the percentage of time during which the system capacity falls below a certain threshold, $P\left(C \leq C_{t h}\right)$. In this study, the Shannon-Hartley formula is used for the calculation:

$$
C=B \log _{2}(1+S N R)
$$

where $B$ is the system bandwidth and SNR represents the Signal to Noise Ratio in linear terms; Equation (1) refers to single links.

In the case of multiple stations in SIMO configuration and taking into account that the receiver has perfect knowledge of the channel, the Maximal Ratio Combining (MRC) scheme can be employed yielding the following capacity [39]:

$$
C_{\text {SIMO }}=B \log _{2}\left(1+\mathbf{h h}^{H}\right)
$$

where $\mathbf{h}=\left[h_{1}, \ldots, h_{M_{R}}\right]$ is the $M_{R} \times 1$ vector of the SIMO channel for the $M_{R}$ receiving antennas. The superscript $H$ refers to the transpose conjugate of the channel vector. The squared measure of the elements in vector $\mathbf{h}$ is given by:

$$
\left|h_{i}\right|^{2}=S N R_{C S, i} 10^{-A_{i} / 10}
$$

where $S N R_{C S, i}$ is the SNR in clear-sky conditions for the link between the satellite and the $i$ receiver's antenna and $A_{i}$ is the rain attenuation induced in the same link. Therefore, (2) becomes:

$$
C_{\text {SIMO }}=B \log _{2}\left(1+\sum_{i=1}^{M_{R}} S N R_{C S, i} 10^{-A_{i} / 10}\right)
$$

Regarding the MISO scheme, it is well-known that capacity cannot be enhanced unless the transmitter has knowledge of the channel conditions [17]; when the channel conditions are known or can be acquired, the signal can be preprocessed before being transmitted. The precoding vector to be used shall then be equal to $[39,40]$ :

$$
\mathbf{w}=\sqrt{M_{T}} \frac{\mathbf{h}^{H}}{\sqrt{\sum_{i=1}^{M_{T}}\left|h_{i}\right|^{2}}}
$$

where $\mathbf{h}=\left[h_{1}, \ldots, h_{M_{T}}\right]$ is the channel vector for the $M_{T}$ transmitting antennas and the squared measure of every element vector, i.e., $\left|h_{i}\right|^{2}$ is given by:

$$
\left|h_{i}\right|^{2}=\frac{S N R_{C S, i}}{M_{T}} 10^{-A_{i} / 10}
$$

where the clear-sky SNR is divided by $M_{T}$ in order to keep the total transmitted power at the same level as in the SISO case. The signal is multiplied with the vector $\mathbf{w}$ before being transmitted and according to (5) the highest power ratio shall occur at the link experiencing the least attenuation. The capacity is given by [39,40]:

$$
\begin{aligned}
C_{\text {MISO }} & =B \log _{2}(1+\mathbf{h w}) \\
& =B \log _{2}\left(1+\sum_{i=1}^{M_{T}} S N R_{C S, i} 10^{-A_{i} / 10}\right)
\end{aligned}
$$




\section{Dual-Site Micro-Scale Diversity Scheme}

\subsection{Experimental Data-Bank}

Two identical EML ARG 100 rain gauges [41] have been deployed at the NTUA Campus in Athens, Greece $\left(37.97^{\circ} \mathrm{N}, 23.78^{\circ} \mathrm{E}\right)$ measuring rainfall at a separation distance of about $386 \mathrm{~m}$; one of them is placed at the old buildings belonging to the School of Electrical and Engineering while the other one at the new ones. The rain gauges used are of tipping bucket type and are pre-calibrated with a measurement resolution of $0.2 \mathrm{~mm}$ of rain per tip; the sampling frequency is set at $1 \mathrm{~Hz}$. Using the recorded rainfall data, rain rate time series in $\mathrm{mm} / \mathrm{h}$ with 1 -min integration time window have been calculated using the methodology presented in [38]. The rain rate data availability for both rain gauges has been $100.00 \%$ for the whole 4-year time period examined in this paper (June 2012-June 2016).

In Figure 2, the obtained rain rate time series with 1-min integration time are presented for both locations for the whole 4-year period. In Figure 3, the Complementary Cumulative Distribution Function (CCDF) of rain rate for each of the locations as well as the jointminimum CCDF for both sites at NTUA Campus are presented.

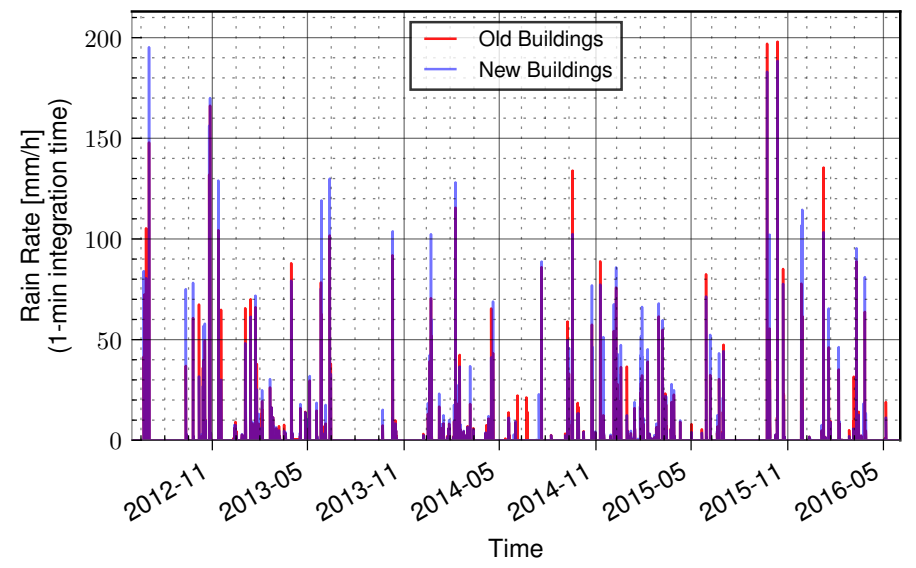

Figure 2. Calculated rain rate time series for the examined period and both locations within the NTUA campus.

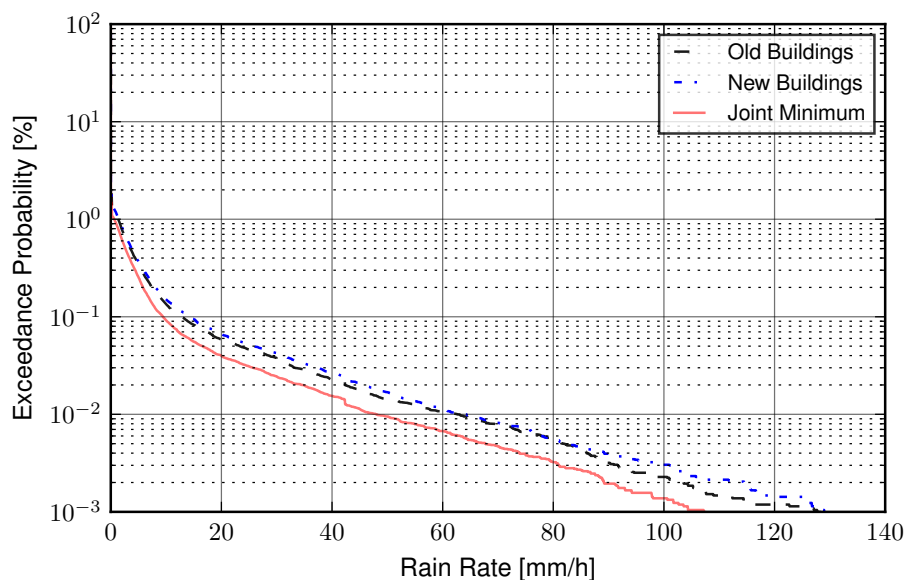

Figure 3. Cumulative distribution of rain rate for the two locations at NTUA campus and the joint-minimum CCDF (solid line).

\subsection{Emulated Joint Rain Attenuation Statistics}

Although the deployment of actual satellite stations to perform measurements has always been preferred over any simulation methods, it is highly unlikely that one would attempt it without having a reliable set of simulated results to perform a first assessment; this is where the benefit of merely using rain gauges, easier to procure and install than satellite stations becomes apparent. Rain attenuation time series can then be generated 
using the Synthetic Storm Technique (SST) with a two-layer model having as input the rain rate time series obtained via the rain gauges [34]. The SST is a physical-mathematical propagation modeling tool based on the Taylor hypothesis in order to translate the rain rate time series into the spatial domain and then calculate the rain attenuation time series on the slant path. Should rainfall rate data be available, the SST must be the preferred choice over any other statistical methodology/theory in order to evaluate the system performance-the application of any of the latter methodologies could yield very questionable results in real applications, especially for low probabilities. To apply the SST, the average storm speed is required along the slant path; for Athens, a value of $10.6 \mathrm{~m} / \mathrm{s}$ is assumed based on meteorological records. In any case, long-term statistics seem to be insensitive to its precise value [40].

The two-layer model is defined in the vertical path and is used to capture the melting layer effects. For the conversion of rain rate $R$ in $\mathrm{mm} / \mathrm{h}$ to the specific rain attenuation $A_{0}(\mathrm{~dB} / \mathrm{km})$, the classic power-law relationship $A_{0}=k R^{b}$ is used. The parameters $k, b$ are calculated from the ITU-R. P. 838 [42] for Layer A (raindrops at $20^{\circ} \mathrm{C}$ ), while the ones in $[37,40]$ are used for Layer B (melting hydrometeors at $0^{\circ} \mathrm{C}$ ).

In Figure 4, the 4-year rain attenuation time series emulated using SST are presented for the two station locations operating at a frequency of $19.701 \mathrm{GHz}$, i.e., a Ka-band downlink. The elevation angle of the links is $45.98^{\circ}$, chosen based on the actual elevation angle (median value) for the geostationary, inclined-orbit satellite Alphasat at 25.0 E [43] as observed from the particular sites. In Figure 5, the CCDF of rain attenuation is given at Ka-band for the two stations, while the CCDF of the joint-minimum rain attenuation is also presented referring to an idealized site diversity scenario; the joint-minimum rain attenuation across $N$ links is

$$
A_{\text {joint }}(t)=\min \left\{A_{1}(t), \ldots, A_{N}(t)\right\}
$$

A gain of almost $2 \mathrm{~dB}$ can be achieved for up to $0.01 \%$ of the time in this idealized micro-scale site diversity case. In Figures 6 and 7 the rain attenuation time series and CCDFs are shown for a Q-band downlink (39.402 GHz), respectively. A gain of $3 \mathrm{~dB}$ for up to $0.1 \%$ of the time can be achieved while a gain of $5 \mathrm{~dB}$ for up to $0.01 \%$ of the time also seems feasible as indicated by the joint-minimum CCDF in Figure 7.

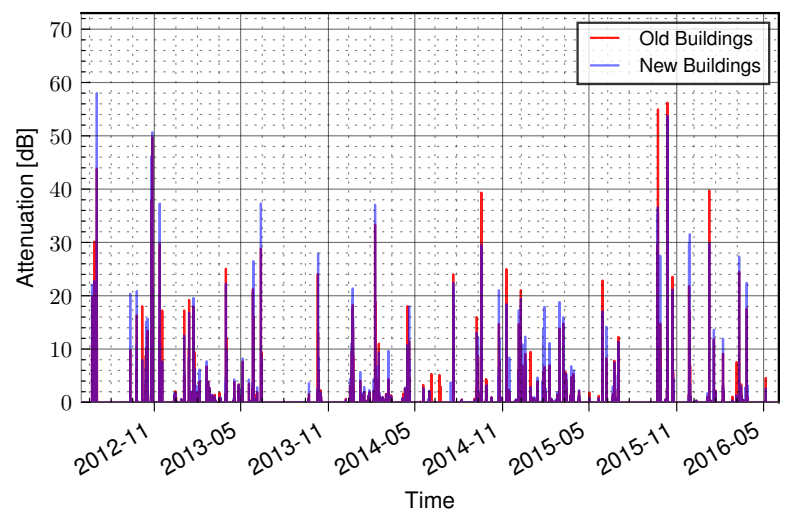

Figure 4. Emulated rain attenuation time series obtained by applying the SST for the two locations at 19.701 GHz, Ka-band downlink. 


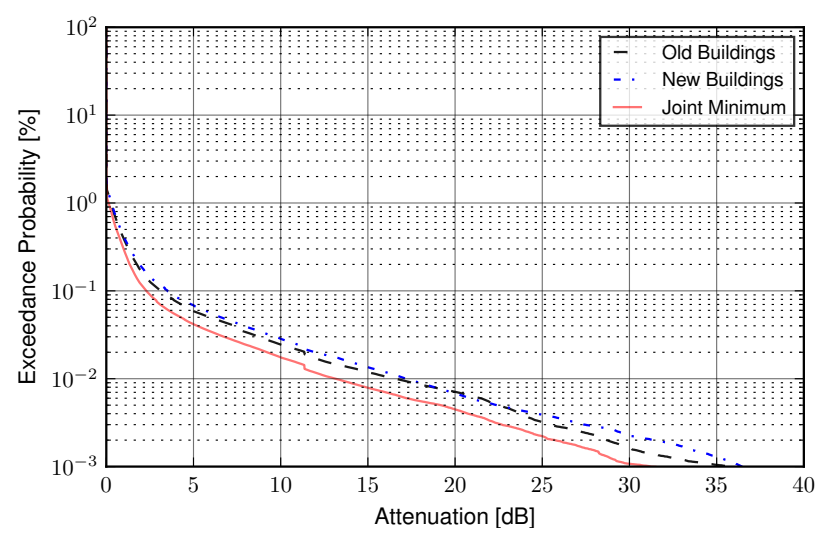

Figure 5. CCDF of rain attenuation for the two locations along with the CCDF of the joint-minimum rain attenuation at $19.701 \mathrm{GHz}, \mathrm{Ka}-$ band downlink.

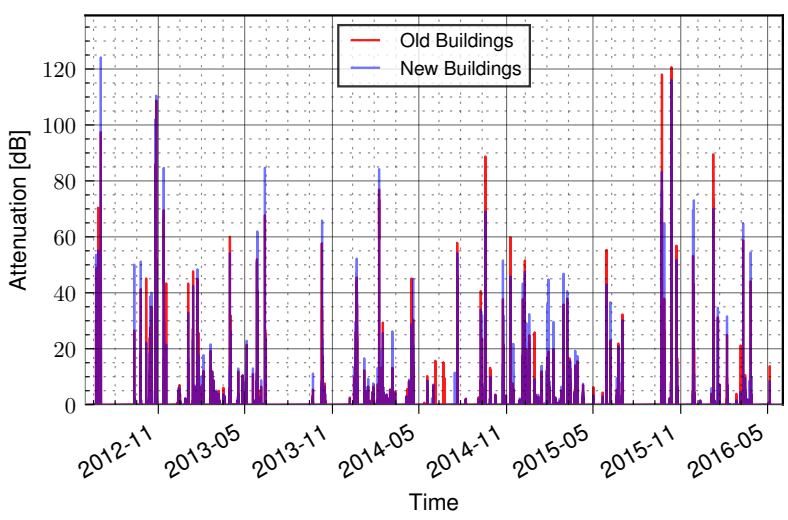

Figure 6. Emulated rain attenuation time series obtained by applying the SST for the two locations at 39.402 GHz, Q-band downlink.

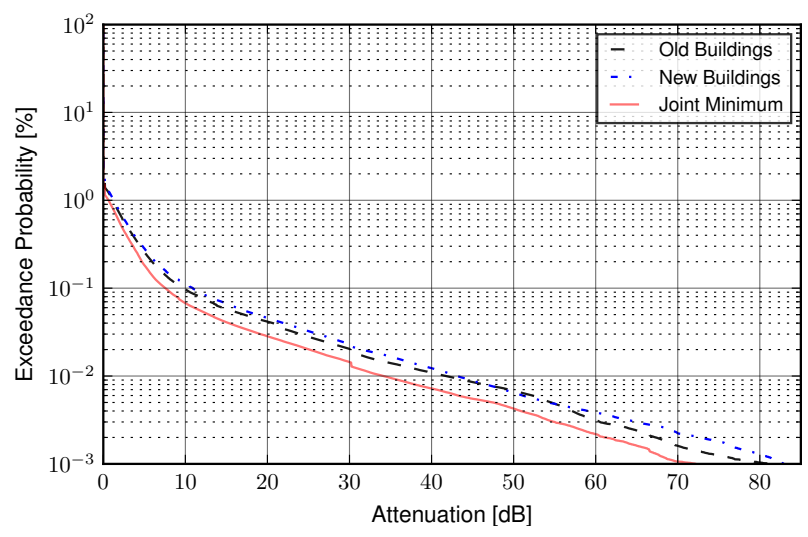

Figure 7. CCDF of rain attenuation for the two locations along with the CCDF of the joint-minimum rain attenuation at $39.402 \mathrm{GHz}$, Q-band downlink.

\subsection{Capacity Evaluation}

In this section, the capacity of a dual MISO-SIMO system is evaluated for a dual-site micro-scale diversity scenario. Using the rain attenuation time series generated using the SST, the capacity time series were obtained using (4) for the SIMO systems and (7) for the MISO ones.

For the SIMO scenario, results are obtained for $19.701 \mathrm{GHz}$ (Ka-band downlink) as well as $39.402 \mathrm{GHz}$ (Q-band downlink). The clear-sky SNR is considered equal to $30 \mathrm{~dB}$. 
In Figure 8, the resulting outage capacity is shown for the SISO and the SIMO system for both Ka-band and Q-band links. It is observed that in clear-sky conditions the microscale system can effectively deliver $11 \mathrm{~b} / \mathrm{s} / \mathrm{Hz}$, while the SISO one $10 \mathrm{~b} / \mathrm{s} / \mathrm{Hz}$. This gain arises from the use of multiple antennas at the receiver, thus proportionally increasing the SNR (also referred to as array gain). As the attenuation increases (lower exceedance probabilities) the micro-scale scheme still offers a gain, e.g., at $0.1 \%$ of the time the capacity for the SISO link is $9 \mathrm{~b} / \mathrm{s} / \mathrm{Hz}$ while for the SIMO one is $10 \mathrm{~b} / \mathrm{s} / \mathrm{Hz}$ regarding the Ka-band downlink case.

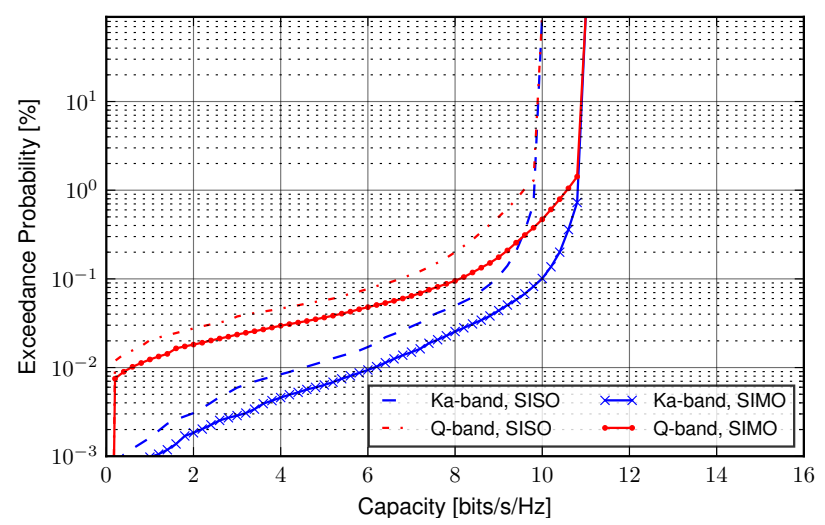

Figure 8. Downlink outage capacity statistics for SISO and SIMO systems operating at Ka- (blue lines) and Q-band (red lines), respectively, for a clear-sky SNR value of $30 \mathrm{~dB}$.

For the uplink, i.e., the MISO case, the outage capacity is shown in Figure 9 for various values of $S N R_{C S}$. The operating frequency is assumed at $29.881 \mathrm{GHz}$, i.e., Ka-band uplink. The gain, also evident in this case, increases with increasing attenuation, i.e., in low probabilities; this is attributed to the precoding vector used in the MISO system. As a matter of fact, in clear-sky conditions all the signals are transmitted with the same power and equal to the power of a SISO link divided by the number of transmitting antennas; it is therefore apparent why there is no gain in clear sky conditions. On the other hand, when attenuation increases, from (5), the highest power ratio will occur at the link experiencing the least attenuation. Therefore, a gain of up to $50 \%$ could be achieved for low probabilities as observed in Figure 9.

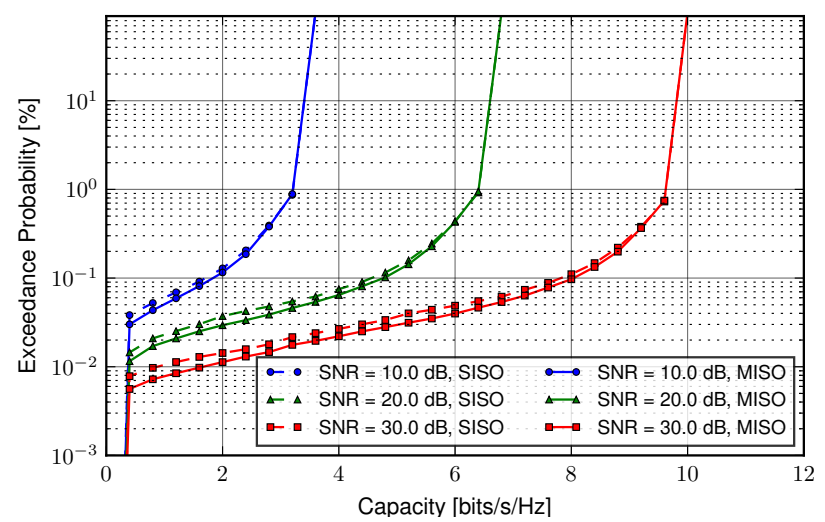

Figure 9. Uplink outage capacity statistics for SISO and MISO systems operating at Ka-band for clear-sky SNR values equal to 10, 20 and $30 \mathrm{~dB}$.

\section{Multi-Site Micro-Scale Diversity Scheme}

In this section, a methodology to generate rain attenuation time series spatially correlated for multiple sites based on Copulas functions is presented. It is generally uncommon for micro-scale satellite communication systems to simultaneously perform in situ beacon measurements. Therefore, the use of a synthesizer to generate rain attenuation time series 
and then calculate the capacity time series for multiple spatially separated links could be of significant importance.

In the proposed model, the synthesizer is based on the assumption that joint rain attenuation statistics in the temporal and the spatial domain are modeled using Gaussian copulas [34,36]. The advantages of using Gaussian copulas to model the joint statistics in the spatial domain are that they are simple to apply and can easily be extended for multiple spatially separated links. Moreover, the synthesizer based on copula functions does not require the proof of any assumption regarding the distribution of the rain attenuation induced on a particular single link [36].

\subsection{Multi-Dimensional Copulas Based Synthesizer}

In this subsection a new multi-dimensional rain attenuation synthesizer is presented, extending the methodology presented in [18], accounting for multiple links used in diversity and multi-hop scenarios. The copula functions are used to couple the single cumulative distributions and to ultimately calculate the joint cumulative distribution. Given that the Cumulative Distribution Functions (CDFs) of dependent random variables are denoted as $u_{i}, i=1, \ldots, n$, the joint statistics can be calculated through copula functions. The Gaussian copula is given by [35]:

$$
P(\mathbf{u})=\boldsymbol{\Phi}_{R}^{n}\left(\boldsymbol{\Phi}^{-1}\left(u_{1}\right), \ldots, \boldsymbol{\Phi}^{-1}\left(u_{n}\right)\right)
$$

where $P(\mathbf{u})$ is the joint CDF of the dependent random variables, $\boldsymbol{\Phi}_{R}^{n}$ is the CDF of a multivariate normal distribution with zero mean and correlation matrix given by $\mathbf{R}$ and $\boldsymbol{\Phi}^{-1}$ is the inverse CDF of a normal distribution with zero mean and unity variance. In (9), the variables $u_{i}, i=1, \ldots, n$, refer to the univariate CDFs of rain attenuation for the multiple links in the case of spatially-joint statistics or to the univariate CDFs of rain attenuation for different time delays in the case of joint statistics in the temporal domain. In order to introduce different notations for the spatial and temporal dependence the Copula function, the correlation matrix of the Gaussian copula and the CDFs of rain attenuation for spatial joint statistics are denoted as: $P_{S P}, \mathbf{R}_{S P}$ and $u^{l}, l=1, \ldots, n$ for $n$ different links, respectively. For the temporal joint statistics, the Copula function is denoted as $P_{T D}$, the correlation matrix as $\mathbf{R}_{T D}$ and the CDF of rain attenuation on different time delays as $u_{j}, j=1, \ldots, K$, with $j$ being the different time delays. Therefore, the CDF of rain attenuation at link $l$ and at time instance $j$ is denoted as $\mathbf{u}_{j}^{l}$. Equation (9) can therefore be used to describe the joint statistics of rain attenuation in the spatial and the temporal domain using different correlation matrices. In [36], an expression for the calculation of the elements of the correlation matrix $\mathbf{R}_{T D}$ in order to generate time series is given; in [34] an expression for the calculation of the elements of the correlation matrix $\mathbf{R}_{S P}$ is given for multiple spatially separated links. It should be noted, however, that the expression derived in [34] is based on measurements with a separation distance of $1.7 \mathrm{~km}$ and higher.

In Figure 10, the joint CCDF calculated from the emulated time series as explained in Section III is shown versus the one obtained using the Gaussian copulas; the copula parameter used was equal to 0.971 . The fitted Gaussian copula exhibits very good performance in modeling the joint statistics for this small separation distance. The copula parameter as proposed in [34] for the same distance, i.e., $386 \mathrm{~m}$, gives a value of 0.9928 , however, as already mentioned, the proposed parameter in [34] is derived using data from site diversity experiments with separation distance higher than $1.7 \mathrm{~km}$. Therefore, in our case the copula parameter is extended using a linear relationship between the copula parameter and the distance as shown below for distances between $386 \mathrm{~m}$ and $1.7 \mathrm{~km}$ :

$$
\rho_{S P}=-0.214 d+1.054
$$

where $0.386 \leq d \leq 1.7$ and $d$ is in $\mathrm{km}$. Moreover, the proposed expression in (10) has been used to reproduce the experimental data in short scale site diversity in [19]. Therefore it is reasonable that a global relationship for the correlation coefficient for small distances may 
be derived based on Equation (10) with small refinements obtained through experimental data.

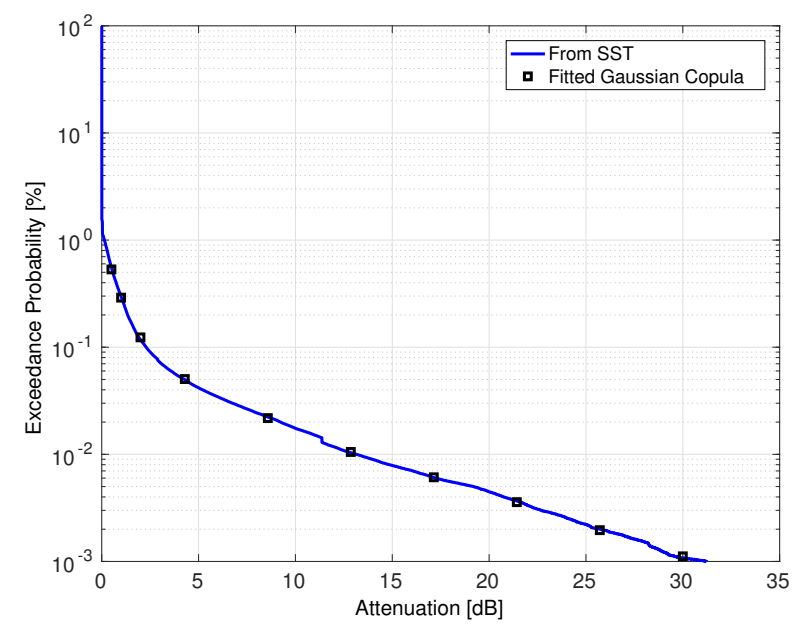

Figure 10. Joint CCDF of rain attenuation calculated from the minimum of the time series using SST vs. the fitted Gaussian copula for $19.701 \mathrm{GHz}$, i.e., Ka-band downlink.

In order to generate rain attenuation time series zero mean and unity variance Gaussian random samples for each of the links are first generated, correlated in the time domain using the correlation matrix $\mathbf{R}_{T D}$. These samples actually correspond to samples of $\boldsymbol{\Phi}^{-1}\left(\mathbf{u}_{j}^{l}\right)$ which refer to time series with only the temporal correlation having been taken into account. Up to this point, the spatial correlation has not been considered, as these time series are produced independently for each link. Then for every time instance $j$ the vector $\mathbf{F}_{j}=\left[\boldsymbol{\Phi}^{-1}\left(\mathbf{u}_{j}^{1}\right), \ldots, \boldsymbol{\Phi}^{-1}\left(\mathbf{u}_{j}^{l}\right), \ldots, \boldsymbol{\Phi}^{-1}\left(\mathbf{u}_{j}^{n}\right)\right]^{T}$ is multiplied on the right side by the Cholesky decomposition of $\mathbf{R}_{S P}$. It should be noted that the multiplication of $\mathbf{F}$ by the Cholesky decomposition of $\mathbf{R}_{S P}$ will again result into Gaussian random variables. Therefore, on every given time instance $j$ the random variables $\boldsymbol{\Phi}^{-1}\left(\mathbf{u}_{j}^{l}\right)$ are now correlated also in the spatial domain. In order to generate time series of rain attenuation the CDF of $\boldsymbol{\Phi}^{-1}\left(\mathbf{u}_{j}^{l}\right)$ has to be calculated first, considering normal zero mean and unity variance distribution in order to obtain $\mathbf{u}_{j}^{l}$; then the inverse CDF of rain attenuation distribution is calculated on the values of $\mathbf{u}_{j}^{l}$. The step-by-step algorithm for generating rain attenuation time series of duration $T$ with sampling time $t_{s}$ for $n$ links is as follows:

1. Calculate the number of samples $K$ for the time series corresponding to each link, $K=T / t_{s}$;

2. Generate $K$ samples of Gaussian random variables with zero mean and covariance matrix $\mathbf{R}_{T D}$;

3. Repeat step $2 n$ times, equal to the number of links;

4. From the samples created in steps 2 and 3 , create the samples of vectors $\mathbf{F}_{j}$ considering the samples from the different links referring to the same time instance $j$;

5. Multiply on the right side every sample of the vector $\mathbf{F}_{j}$ with the Cholesky decomposition of $\mathbf{R}_{S P}$;

6. $\quad$ Create the matrix $\mathbf{U}=\left[\mathbf{F}_{1}, \ldots, \mathbf{F}_{K}\right]^{T}$;

7. Calculate on every element the normal CDF;

8. On every element of each line $i$ calculate the inverse CDF of rain attenuation induced on link $i$;

9. Every line of the matrix created in step 8 should now correspond to the rain attenuation time series on each link.

An example of the time series generated is shown in Figure 11, where three stations are considered to form an equilateral triangle with sides equal to $386 \mathrm{~m}$. The joint-minimum of the rain attenuation time series is also shown in the same figure. To validate the above 
methodology before applying it to the multi-dimensional case, the joint CCDF of rain attenuation as obtained from the emulated data (i.e., using the SST) vs. simulated data using the copula synthesizer for two links is presented in Figure 12; from the results obtained it can be concluded that the synthesized data are in excellent accordance with the emulated ones. The second order statistics (fade durations, fade slopes, cross correlation etc.) are not investigated since they are beyond the scope of this contribution.

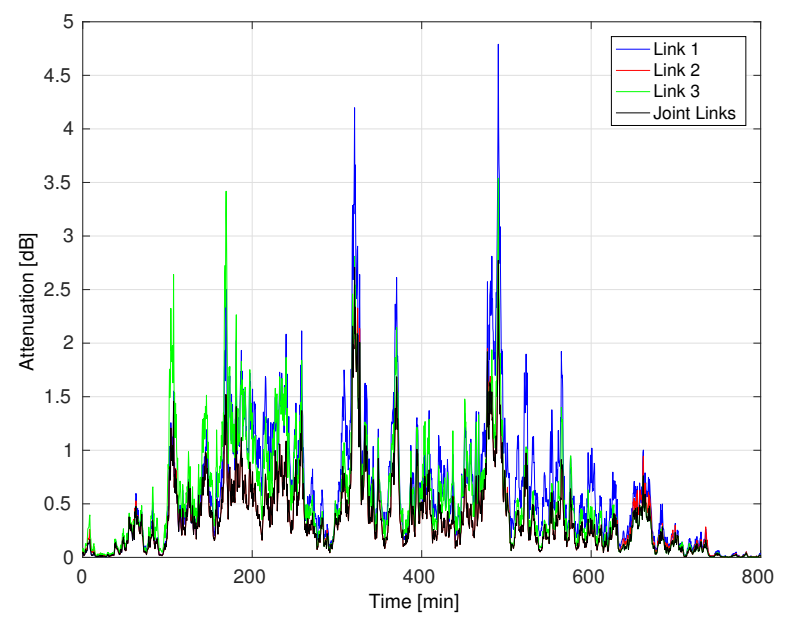

Figure 11. Simulated rain attenuation time series using the proposed copula synthesizer for three ground stations in Athens at $19.701 \mathrm{GHz}$, i.e., Ka-band downlink.

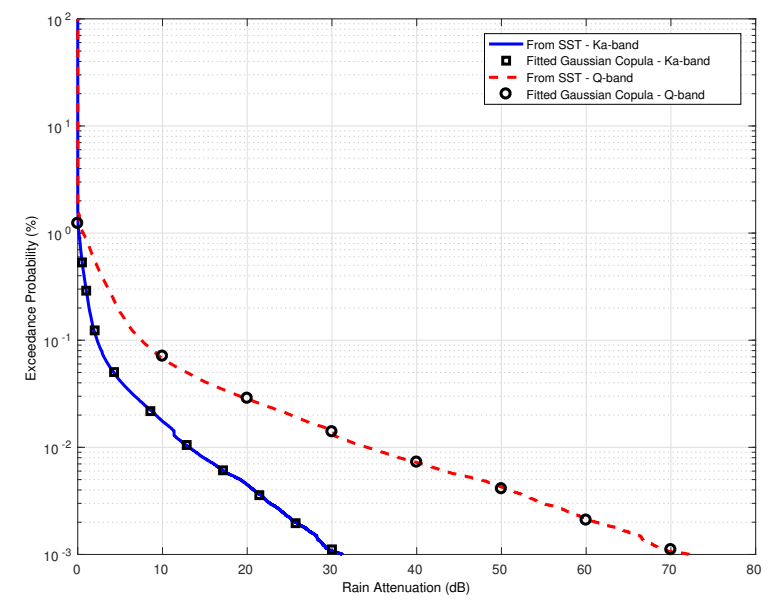

Figure 12. Joint CCDF of rain attenuation from the multi-dimensional synthesizer vs. the emulated time series for systems operating at Ka- and Q-band.

\subsection{Numerical Results}

Using the multi-dimensional synthesizer proposed in the previous subsection, capacity time series can be generated for SIMO and MISO satellite communication systems. In Figure 13 time series of downlink capacity for a single link and a SIMO system with three ground stations are shown. The use of such a methodology to create capacity time series bears the advantage that the cumulative statistics of rain attenuation from either measurements, emulated time series or any other prediction method can be utilized, including numerical techniques, such as the prediction by ITU-R. P. 618 [44] or any other well-established distribution used for the modeling the rain attenuation such as the Weibull, the log-normal, etc. [45-47]. In this paper, the cumulative distribution of rain attenuation derived from the SST was used directly; the frequency was assumed equal to $19.701 \mathrm{GHz}$ and the elevation angle equal to $45.98^{\circ}$ as in the previous section. 
The outage capacity statistics for the downlink SISO and SIMO cases for 2, 3 and 4 receiving antennas are shown in Figure 14. Regarding the two station scenario, the stations are placed $386 \mathrm{~m}$ apart while in the three station case, the stations form an equilateral triangle comprising of $386 \mathrm{~m}$ sides. Finally, the four stations corresponding to the last scenario are placed in a square grid with $386 \mathrm{~m}$ sides. It is apparent that the gain introduced in the system compared to the single-link case is quite pronounced and as such, micro-scale site diversity systems may provide a considerable diversity gain for both clear-sky and rainy conditions. More presicely, a capacity gain of about $40 \%$ can be observed for $M_{R}=2$ and $82 \%$ for $M_{R}=4$ at $0.01 \%$ of the time (i.e., $10^{-4}$ probability). In Figure 15, the outage capacity for $1 \%$ of the time is shown for the Ka-band downlink SISO and SIMO cases considering various clear-sky SNR values; a gain in the order of $50 \%$ for four stations is observed.

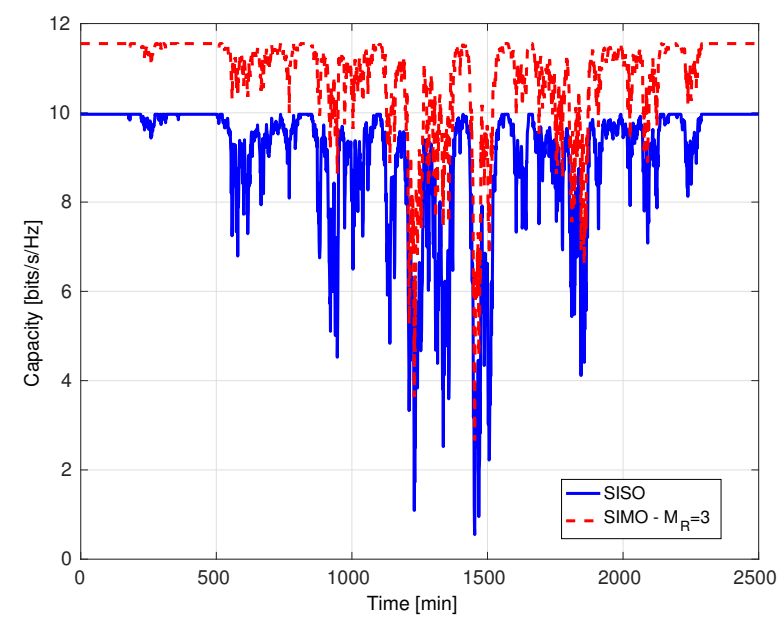

Figure 13. Capacity time series for SISO and SIMO with three stations for the Ka-band downlink at $19.701 \mathrm{GHz}$ using the copula synthesizer.

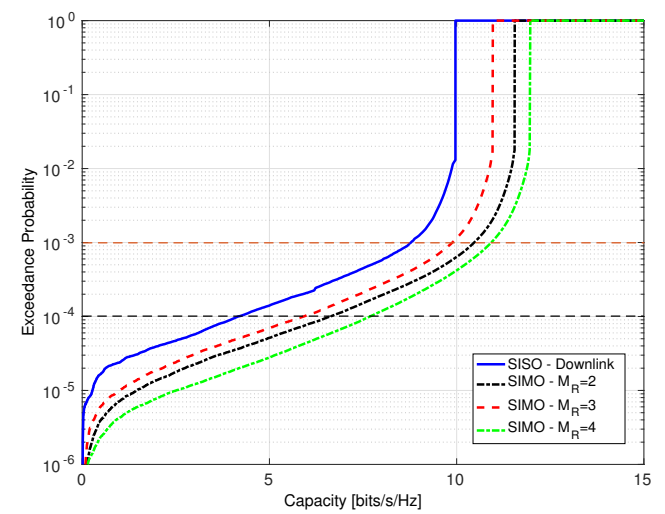

Figure 14. Ka-band (19.701 GHz) downlink outage capacity statistics for the SISO and the SIMO case with 2, 3 and 4 stations using the copula synthesizer. 


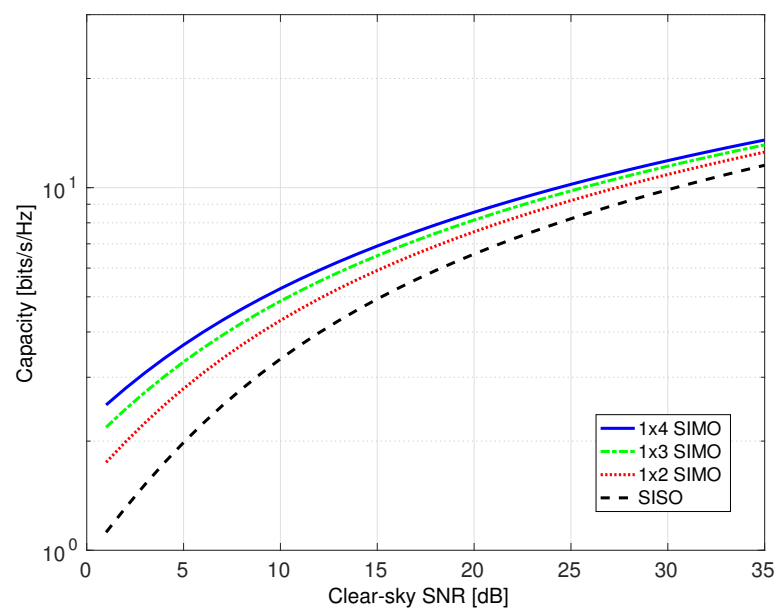

Figure 15. Downlink capacity vs. SNR for Ka-band at $19.701 \mathrm{GHz}$ using SISO and SIMO configurations in clear-sky conditions achieved at $1 \%$ of the time.

For the case of a MISO system, the uplink from a user terminal with multiple antennas is examined. In order to have a fair comparison with the SISO system, the uplink power transmitted through each of the MISO system's antennas is divided by their number as also explained in Section III. Using the multi-dimensional synthesizer proposed in the previous subsection, capacity time series are generated for MISO satellite communication systems using again 2, 3 and 4 ground stations $\left(M_{T}\right)$ and geometries similar to the ones used in the SIMO system. Since MISO techniques refer to the uplink/return-link of a user's terminal, the operating frequency has been chosen at $29.881 \mathrm{GHz}$. As in the previous numerical results, the CDF of rain attenuation calculated from the SST is used for the generation of rain attenuation time series. The uplink outage capacity is shown in Figure 16 for the three MISO cases and the corresponding SISO links; as in the case of downlink, a gain is also observed in the micro-scale site diversity system when the MISO technique is employed. At $0.01 \%$ of the time (i.e., $10^{-4}$ probability), the achievable capacity gain almost reaches $100 \%$ for $M_{T}=2$ and up to $190 \%$ for $M_{T}=4$, respectively. The diversity gain is further increased with increasing the number of antennas. Due to the fair comparison in terms of transmitted power, the capacity in clear-sky conditions remains the same, however, the use of multiple transmitting antennas under rainy conditions offers a substantial gain compared to the SISO case. Increasing the number of transmitting stations even further, a higher gain under rain is to be observed.

As a final remark, the difference between the MISO and SIMO system is the gain at higher time percentages where SIMO clearly outperforms a corresponding SISO architecture.

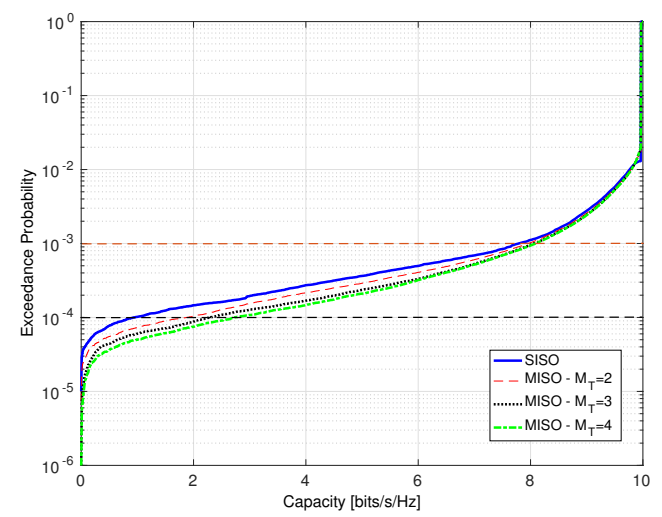

Figure 16. Uplink outage capacity statistics for the SISO and the MISO case with 2, 3 and 4 stations using the copula synthesizer. 


\section{Conclusions}

In this paper, SIMO and MISO micro-scale site diversity systems are evaluated in terms of capacity and compared to the conventional SISO case for both downlink and uplink scenarios, respectively. Using the 4-year experimental rainfall data-bank from two stations at NTUA campus, the SST is used to obtain rain attenuation time series. From the rain attenuation time series the outage capacity is calculated assuming receive (SIMO) and transmit (MISO) diversity schemes with perfect channel state knowledge at the user terminal; a gain seems to always be present for both cases and at both Ka- and Q-band. A multi-dimensional time series synthesizer based on Gaussian copulas is proposed for the generation of spatio-temporally dependent time series of rain attenuation. Using the rain attenuation time series from the SST, the parameter of Gaussian copula is calculated for micro-scale site diversity systems. Making use of the proposed synthesizer, micro-scale site diversity systems with multiple ground stations are evaluated, concluding that a considerable gain is always present for both clear-sky and rainy conditions. Experimental propagation campaigns for micro-scale site diversity scenarios must be conducted in order to validate the presented results.

It has been shown that even small separation distances in the order of a few hundred meters can significantly improve system performance. Micro-scale site diversity systems can therefore be exploited by enterprise users to increase data rates, ensure high system availability and therefore allow for trouble-free operation of time-critical, high data volume applications. Part of the pending future work on the subject is the assessment of the channel capacity within the framework of real modulation schemes (e.g., QAM) comparing the results with the ones based solely on the Shannon's Gaussian channel.

Author Contributions: Conceptualization, A.Z.P. and A.D.P.; Formal analysis, A.Z.P. and A.D.P.; Investigation, A.Z.P.; Methodology, A.Z.P., A.D.P. and C.I.K.; Software, A.Z.P. and C.I.K.; Supervision, A.D.P.; Validation, A.Z.P.; Visualization, A.Z.P. and C.I.K.; Writing-original draft, A.Z.P. and A.D.P.; Writing-review and editing, A.Z.P. and A.D.P. All authors have read and agreed to the published version of the manuscript.

Funding: This research received no external funding.

Conflicts of Interest: The authors declare no conflict of interest.

\section{References}

1. Sharma, S.K.; Chatzinotas, S.; Arapoglou, P.D. (Eds.) Satellite Communications in the 5G Era; Telecommunications, Institution of Engineering and Technology: London, UK 2018.

2. Völk, F.; Liolis, K.; Corici, M.; Cahill, J.; Schwarz, R.T.; Schlichter, T.; Troudt, E.; Knopp, A. Satellite Integration into 5G: Accent on First Over-The-Air Tests of an Edge Node Concept with Integrated Satellite Backhaul. Future Internet 2019, 11, 193. [CrossRef]

3. Gupta, A.; Jha, R.K. A Survey of 5G Network: Architecture and Emerging Technologies. IEEE Access 2015, 3, 1206-1232. [CrossRef]

4. Evans, B.G. The role of satellites in 5G. In Proceedings of the 2014 7th Advanced Satellite Multimedia Systems Conference and the 13th Signal Processing for Space Communications Workshop (ASMS/SPSC), Livorno, Italy, 8-10 September $2014 ;$ pp. 197-202. [CrossRef]

5. Shi, S.; An, K.; Li, G.; Li, Z.; Zhu, H.; Zheng, G. Optimal Power Control in Cognitive Satellite Terrestrial Networks With Imperfect Channel State Information. IEEE Wirel. Commun. Lett. 2018, 7, 34-37. [CrossRef]

6. Wang, C.; Bian, D.; Shi, S.; Xu, J.; Zhang, G. A Novel Cognitive Satellite Network With GEO and LEO Broadband Systems in the Downlink Case. IEEE Access 2018, 6, 25987-26000. [CrossRef]

7. Morel, C.; Arapoglou, P.D.; Angelone, M.; Ginesi, A. Link adaptation strategies for next generation satellite video broadcasting: A system approach. IEEE Trans. Broadcast. 2015, 61, 603-614. [CrossRef]

8. Nakazawa, S.; Nagasaka, M.; Tanaka, S.; Shogen, K. A method to control phased array antenna for rain fading mitigation of 21-GHz band broadcasting satellite. In Proceedings of the Fourth European Conference on Antennas and Propagation, Barcelona, Spain, 12-16 April 2010; pp. 1-5.

9. Lei, J.; Vázquez-Castro, M.Á. Multibeam satellite frequency/time duality study and capacity optimization. J. Commun. Netw. 2011, 13, 472-480. [CrossRef]

10. Vasavada, Y.; Gopal, R.; Ravishankar, C.; Zakaria, G.; BenAmmar, N. Architectures for next generation high throughput satellite systems. Int. J. Satell. Commun. Netw. 2016, 34, 523-546. [CrossRef] 
11. Katona, Z.; Clazzer, F.; Shortt, K.; Watts, S.; Lexow, H.P.; Winduratna, R. Performance, cost analysis, and ground segment design of ultra high throughput multi-spot beam satellite networks applying different capacity enhancing techniques. Int. J. Satell. Commun. Netw. 2016, 34, 547-573. [CrossRef]

12. Roumeliotis, A.J.; Kourogiorgas, C.I.; Panagopoulos, A.D. Dynamic Capacity Allocation in Smart Gateway High Throughput Satellite Systems Using Matching Theory. IEEE Syst. J. 2019, 13, 2001-2009. [CrossRef]

13. Matricciani, E. A method to achieve clear-sky data-volume download in satellite links affected by tropospheric attenuation. Int. J. Satell. Commun. Netw. 2016, 34, 713-723. doi:10.1002/sat.1126. [CrossRef]

14. Matricciani, E. Probability distributions of rain attenuation obtainable with linear combining techniques in space-to-Earth links using time diversity. Int. J. Satell. Commun. Netw. 2018, 36, 220-237. doi:10.1002/sat.1214. [CrossRef]

15. Kanatas, A.; Panagopoulos, A.D. Radio Wave Propagation and Channel Modeling for Earth-Space Systems; CRC Press: Boca Raton, FL, USA, 2016; Chapter 2.

16. Crane, R.K. Propagation Handbook for Wireless Communication System Design; CRC Press: Boca Raton, FL, USA, 2003.

17. Panagopoulos, A.D.; Arapoglou, P.D.M.; Cottis, P.G. Satellite communications at Ku, Ka, and V bands: Propagation impairments and mitigation techniques. IEEE Commun. Surv. Tutor. 2004, 6, 2-14. [CrossRef]

18. Matricciani, E. Micro scale site diversity in satellite and tropospheric communication systems affected by rain attenuation. Space Commun. 2003, 19, 83-90.

19. Acosta, R.J. Special effects: Antenna wetting, short distance diversity and depolarization. Online J. Space Commun. 2002, 2, 1-9.

20. Enjamio, C.; Vilar, E.; Ndzi, D.; Perez-Fontán, F. Short-scale diversity in a dynamic rain fade environment. Int. J. Satell. Commun. Netw. 2005, 23, 143-152. [CrossRef]

21. Kourogiorgas, C.; Panagopoulos, A.D.; Livieratos, S.N.; Chatzarakis, G.E. Pico-scale Dynamic Diversity Gain Evaluation in Broadband Satellite Communication Systems. In Proceedings of the EuCAP 2015, Lisbon, Portugal, $12-17$ April 2015.

22. Paulraj, A.; Nabar, R.; Gore, D. Introduction to Space-Time Wireless Communications; Cambridge University Press: Cambridge, UK, 2003.

23. Kyrgiazos, A.; Evans, B.G.; Thompson, P. On the gateway diversity for high throughput broadband satellite systems. IEEE Trans. Wirel. Commun. 2014, 13, 5411-5426. [CrossRef]

24. Rossi, T.; De Sanctis, M.; Maggio, F. Evaluation of outage probability for satellite systems exploiting smart gateway configurations. IEEE Commun. Lett. 2017, 21, 1541-1544. [CrossRef]

25. Jeannin, N.; Castanet, L.; Radzik, J.; Bousquet, M.; Evans, B.; Thompson, P. Smart gateways for terabit/s satellite. Int. J. Satell. Commun. Netw. 2014, 32, 93-106. [CrossRef]

26. Ventouras, S.; Reeves, R.; Rumi, E.; Perez-Fontan, F.; Machado, F.; Pastoriza, V.; Rocha, A.; Mota, S.; Jorge, F.; Panagopoulos, A.D.; et al. Large scale assessment of Ka/Q band atmospheric channel across Europe with ALPHASAT TDP5: The augmented network. In Proceedings of the 2017 11th European Conference on Antennas and Propagation (EUCAP), Paris, France, 19-24 March 2017; pp. 1471-1475. [CrossRef]

27. Papafragkakis, A.Z.; Panagopoulos, A.D.; Ventouras, S. Combined beacon and noise satellite propagation measurements using software defined radio. In Proceedings of the 2017 11th European Conference on Antennas and Propagation (EUCAP), Paris, France, 19-24 March 2017; pp. 2356-2360. [CrossRef]

28. Digital Video Broadcasting (DVB). Second Generation Framing Structure, Channel Coding and Modulation Systems for Broadcasting, Interactive Services, News Gathering and Other Broadband Satellite Applications; Technical Report; ETSI: Sophia-Antipolis, France, 2005.

29. Digital Video Broadcasting (DVB). Second Generation Framing Structure, Channel Coding and Modulation Systems for Broadcasting, Interactive Services, News Gathering and Other Broadband Satellite Applications, Part II: S2-Extensions (S2-X) EN 302307-2; Technical Report; ETSI: Sophia-Antipolis, France, 2014.

30. Karagiannis, G.A.; Panagopoulos, A.D.; Kanellopoulos, J.D. Multidimensional rain attenuation stochastic dynamic modeling: Application to earth-space diversity systems. IEEE Trans. Antennas Propag. 2012, 60, 5400-5411. [CrossRef]

31. Cheffena, M.; Braten, L.E.; Ekman, T. On the space-time variations of rain attenuation. IEEE Trans. Antennas Propag. 2009, 57, 1771-1782. [CrossRef]

32. Maseng, T.; Bakken, P. A stochastic dynamic model of rain attenuation. IEEE Trans. Commun. 1981, 29, 660-669. [CrossRef]

33. Jeannin, N.; Féral, L.; Sauvageot, H.; Castanet, L.; Lacoste, F. A large-scale space-time stochastic simulation tool of rain attenuation for the design and optimization of adaptive satellite communication systems operating between 10 and $50 \mathrm{GHz}$. Int. J. Antennas Propag. 2012, 2012, 749829. [CrossRef]

34. Kelmendi, A.; Kourogiorgas, C.; Hrovat, A.; Panagopoulos, A.D.; Kandus, G.; Vilhar, A. Prediction of joint rain attenuation statistics induced on earth-satellite multiple site diversity systems using Gaussian copula. IEEE Antennas Wirel. Propag. Lett. 2017, 16, 95-98. [CrossRef]

35. Nelsen, R.B. An introduction to Copulas; Springer Science \& Business Media: London, UK 2007.

36. Kourogiorgas, C.; Kelmendi, A.; Panagopoulos, A.D.; Vilhar, A. On rain attenuation time series generation: A new simple copula-based channel model for satellite slant paths. IEEE Trans. Antennas Propag. 2016, 64, 3206-3211. [CrossRef]

37. Matricciani, E. Physical-mathematical model of the dynamics of rain attenuation based on rain rate time series and a two-layer vertical structure of precipitation. Radio Sci. 1996, 31, 281-295. [CrossRef]

38. Matricciani, E.; Riva, C. The search for the most reliable long-term rain attenuation CDF of a slant path and the impact on prediction models. IEEE Trans. Antennas Propag. 2005, 53, 3075-3079. [CrossRef] 
39. Lo, T. Maximum ratio transmission. IEEE Trans. Commun. 1999, 47, 1458-1461. [CrossRef]

40. Kanellopoulos, S.A.; Panagopoulos, A.D.; Matricciani, E.; Kanellopoulos, J.D. Annual and diurnal slant path rain attenuation statistics in Athens obtained with the synthetic storm technique. IEEE Trans. Antennas Propag. 2006, 54, 2357-2364. [CrossRef]

41. EML Environmental Measurements Limited. ARG100 Rain Gauge User Manual. Available online: http://www.emltd.net/ uploads/1/0/5/1/105135655/um-780-001-arg100.pdf (accessed on 1 August 2021)

42. ITU-R Recommendation P.838-3: Specific Attenuation Model for Rain for Use in Prediction Models; Technical Report; International Telecommunication Union: Geneva, Switzerland, 2005.

43. Paraboni, A.; Riva, C.; Capsoni, C.; Codispoti, G.; Zuliani, L.; Speziale, V.; Falzini, S.; Martellucci, A.; Colzi, E. Description of Alphasat TDP5 Propagation Experiment. In Proceedings of the ESA Workshop “Radiowave Propagation Models, Tools, and Data for Space Systems", Noordwijk, The Netherlands, 3-5 December 2008; pp. 1-4.

44. ITU-R Recommendation P.618-13: Propagation Data and Prediction Methods Required for the Design of Earth-Space Communication Systems; Technical Report; International Telecommunication Union: Geneva, Switzerland, 2017.

45. Boulanger, X.; Feral, L.; Castanet, L.; Jeannin, N.; Carrie, G.; Lacoste, F. A rain attenuation time-series synthesizer based on a dirac and lognormal distribution. IEEE Trans. Antennas Propag. 2013, 61, 1396-1406. [CrossRef]

46. Kanellopoulos, S.A.; Panagopoulos, A.D.; Kourogiorgas, C.I.; Kanellopoulos, J.D. Satellite and terrestrial links rain attenuation time series generator for heavy rain climatic regions. IEEE Trans. Antennas Propag. 2013, 61, 3396-3399. [CrossRef]

47. Kanellopoulos, S.A.; Kourogiorgas, C.I.; Panagopoulos, A.D.; Livieratos, S.N.; Chatzarakis, G.E. Channel model for satellite communication links above $10 \mathrm{GHz}$ based on Weibull distribution. IEEE Commun. Lett. 2014, 18, 568-571. [CrossRef] 